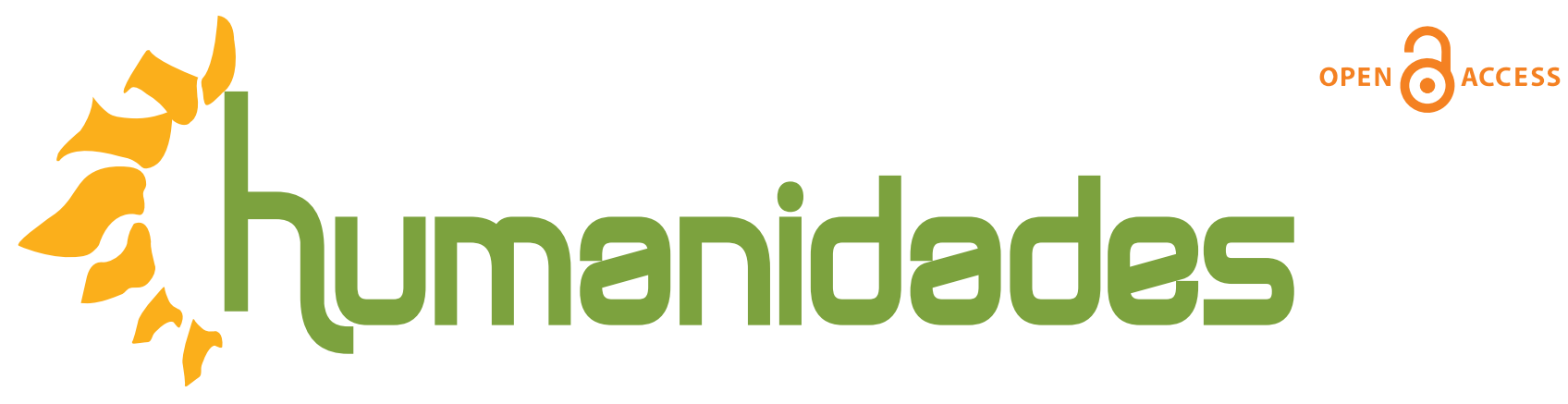

Revista de la Escuela de Estudios Generales, Universidad de Costa Rica

Julio-diciembre, 2019 •Volumen 9, número 2・EISSN 2215-3934・pp. 1-23

Recibido: 01-Febrero-2019 Aceptado: 25-Abril-2019

\title{
Politización mediática de la justicia en Ecuador. Estudio de caso: Ecuavisa
}

DOI: https://doi.org/10.15517/h.v9i2.37659

\section{Kevin Paúl Pullaguari Zaruma}

Máster

Universidad Internacional de Andalucía, España

Correo electrónico: kevin.pullaguari103@alu.uhu.es / kppullaguari@hotmail.com

\section{Ángel Hernando Gómez}

Doctor

Universidad de Huelva, España

Correo electrónico: angel.hernando@dpsi.uhu.es

Todos los derechos reservados. Universidad de Costa Rica. Esta revista se encuentra licencida con Creative Commons. Reconocimiento-NoComercial-SinObraDerivada 3.0 Costa Rica. Correo electrónico: humanidades@ucr.ac.cr/ Sitioweb: http: //revistas.ucr.ac.cr/index.php/humanidades 


\section{Politización mediática de la justicia en Ecuador. Estudio de caso: Ecuavisa}

\section{Resumen}

La politización mediática de la justicia en el contexto ecuatoriano es un tema emergente en Latinoamérica del cual no existe mucha información en el ámbito académicocientífico. Esta investigación aborda esta situación de politización, en un entorno marcado por los medios de comunicación, precisamente, desde el canal Ecuavisa y el poder judicial. La metodología utilizada es el estudio de caso, llevado a cabo mediante la observación no reactiva de nueve noticias, a través de una rejilla de análisis, y la entrevista semiestructurada a dos expertos del tema. Este análisis determina la situación social y democrática del país y crea conciencia sobre el rol que juegan los medios ante estas circunstancias de politización. Ecuavisa es el medio más sintonizado en Ecuador con el $28.02 \%$ de la audiencia, lo que ha generado en los últimos años gran concentración mediática gracias al protagonismo que se ha ganado dicho canal, debido al manejo de la información emitida. El escenario que vive el país muestra una tendencia política determinada a pesar de la imparcialidad-objetividad con la que se pretenden difundir las noticias. Se constató que la información transmitida en la pantalla de Ecuavisa muestra gran intencionalidad de influenciar en el pensamiento-opinión de los ciudadanos.

\section{Media Politicization of Justice in Ecuador. Case Study: Ecuavisa}

\begin{abstract}
The media politicization of justice in the Ecuadorian context is an emergent subject in Latin America from which there is not much information in the academic-scientific field. This investigation addresses this situation for the country, in an environment marked by the media, precisely the channel "Ecuavisa" and the judiciary. The methodology used is the Case Study objectivity with which is intended the empowerment, analyzed through a non-reactive observation, a grid analysis and a semi-structured interview with two experts on the subject. This analysis determines the social and democratic situation of the country and creates awareness about the role the media plays in these situations. The results show that this channel is the most watched and tuned in Ecuador with $28.02 \%$ of audience, which has generated in the recent years a great media concentration through the prominence that has been gained, due to the information published. The scenario that the country is living shows a determined political tendency despite of the impartiality-objectivity with which it's intended to communicate the news. It was found that the information transmitted on its screen shows great intentionality with the aim of influencing the empowerment of citizens' thinking-opinion.
\end{abstract}

Palabras clave: poder judicial, política, medios de comunicación, opinión pública, alfabetización informacional

Keywords:

judicial power, politics, mass media, public opinion, information literacy 


\section{Introducción}

El concepto de "politización mediática" ha recibido considerable atención académica en los últimos años es por eso que, paulatinamente, mediante investigaciones se está dando a conocer cómo los medios de comunicación (MC) sesgan y mediatizan la información, influyendo así, en la politización de las justicias democráticas de los estados, así como también, en el pensamiento u opinión de los ciudadanos.

\footnotetext{
...la información es restringida, manipulada a conveniencia y considerada "material sensible" bajo vigilancia pública; en los sistemas democráticos, por contraste, se considera que los medios actúan bajo el supuesto de independencia respecto al Gobierno, al servicio de la sociedad y, aparentemente, no motivados por intereses económicos espurios, ya que la información es derecho y servicio público al mismo tiempo. (Blesa-Aledo, 2006, p. 93)
}

Por tanto, los medios de comunicación juegan un rol importante en la politización mediática de la justicia, sin embargo, la sociedad en general obvia esta situación. "Es mucho más exacto pensar que las instituciones de los medios son sitios de intereses políticos, profesionales y económicos coexistentes, a veces opuestos, a veces coextensivos. Hablar de "una" lógica de medios es demasiado simplista" (Downey \& Neyazi, 2014, p. 480).

Es importante mencionar que "la política vinculada jurídicamente pierde su ductilidad y flexibilidad, su espontaneidad, la capacidad de reaccionar rápidamente a situaciones de la vida en permanente cambio" (Haas, 2010, p. 369). Pues, este entorno que tiene que ver con los medios, la política y la justicia, es el que está tomando relevancia diariamente, por esa razón, es de vital importancia la alfabetización mediática de la ciudadanía con el objetivo de empoderarla ante este tipo de fenómenos. 
Con base en lo suscrito y en el estudio planteado, este trabajo de investigación pretende analizar el caso del canal televisivo Ecuavisa en la politización mediática de la justicia ecuatoriana, así como la intencionalidad de su información con el público en función de sus intereses. El objetivo es realizar una investigación que contenga datos reales en profundidad, lo cual se efectuará mediante una entrevista semiestructurada a dos expertos y el análisis de la información emitida por dicho medio con la ayuda de una rejilla de investigación web creada por los investigadores.

\subsection{Politización de la justicia}

En los países de América Latina y en muchas nuevas democracias, la reflexión acerca de la relación entre política y derecho ha tomado un nuevo cauce. Después de haber estado centrada en la dependencia del poder judicial respecto del poder político, de la mano de la creciente importancia que adquiere la actividad judicial, inicia un viraje hacia la forma en que el poder judicial interviene y procesa conflictos políticos. En otros términos, la balanza ha comenzado a inclinarse desde la preocupación por la dependencia del poder judicial a la preocupación por la dependencia del poder político de las decisiones judiciales. La tematización de las tan mentadas judicializaciones de la política y politización de la justicia, forma parte de este nuevo impulso (Ansolabehere, 2005, p. 39)

Empero, ¿qué es politización? Según el diccionario de la Real Academia Española de la Lengua (DRAE, 2018) es una acción y efecto de politizar, es decir, dar orientación o contenido político a acciones, pensamientos, etc., que corrientemente, no lo tienen. Por su parte, Medrano (2017) define que la politización supone la injerencia, intromisión e invasión de la política en áreas que le son ajenas y no le competen (desnaturalizándolas y corrompiéndolas). Se trata de un fenómeno de la política: su hipertrofia que la convierte en un cáncer, con nefastas metástasis en el cuerpo social. Fenómeno este, sumamente contagioso y que se propaga con gran facilidad, infectando todo cuanto toca o cae en su radio de acción. Por lo que, si profundizamos en dicho término, podemos notar que la politización no se basa en argumentos de la lógica jurídica sino en la lógica política, pues son los órganos políticos quienes imparten justicia a su beneficio. 
Politización mediática de la justicia en Ecuador...

Y por consecuencia, es probable que los fiscales abandonen su resistencia contra el presidente, lo que deriva la aceptación de cambios, incluso desfavorables que se les impongan (Lee, 2016). Por su parte, Maravall (como se citó en Lee, 2016) sostiene que en la práctica, un Gobierno en ejercicio ha explotado a menudo los poderes de los funcionarios judiciales para objetivos partidistas-proselitistas, como por ejemplo la eliminación de sus oponentes políticos, en muchas democracias modernas.

Por lo tanto, las condiciones y la forma en que se produce la politización de la justicia tienen mucho que ver con la toma de decisiones justificadas empíricamente por la función política, con la finalidad de que sus pretensiones se cumplan a corto o largo plazo, pues es ahí que las leyes deben cumplirse y controlarse a través de la objetividad e imparcialidad. Por ello, son de vital importancia los valores y la ética para no actuar en contra de las leyes que son el marco formal en las que deben basarse las y los que juegan el papel de decisión, para de esa manera no rebasarse cuando se adopta cualquier medida arbitraria.

Bajo la influencia de las doctrinas de los filósofos griegos, desde la antigüedad y a lo largo de muchos siglos, fue communis opinio que la ética debía disciplinar el contenido de la política, como parte de ésta. El poder ejercido bajo este principio no corre peligro de caer en el abuso o la arbitrariedad. (Haas, 2010, p. 368)

En síntesis, la justicia debe seguir siendo un poder autónomo, independiente y democrático con la firme intención de no vulnerar el Estado de derecho de las personas y de los pueblos. En palabras de Ferrero (2009), indistintamente cual sea el caso, la apreciación cuantitativa tiene un valor limitado, incluso irrelevante si se tiene en cuenta el impacto que un trato injusto y brutal tiene sobre una población ignorada por el aparato de poder y que carece de medios para combatir la impunidad del mismo. 


\subsection{Influencia y poder de los MC para mover masas hacia una opinión u otra}

La influencia y poder de los MC para mover masas sociales hacia una opinión u otra ha alcanzado una dimensión única e inigualable. En la medida que los usuarios interactúan con ellos, mediante sus chats abiertos, hashtag (etiqueta), entrevistas en vivo y más; denotando así, sus habilidades, formas de pensar a favor o en contra, entre otras. Por ejemplo, Towner y Muñoz (2016) en su investigación llevaron a cabo un estudio de caso, donde concluyeron cómo la dependencia de ciertos tipos de medios influye en las actitudes políticas y comportamientos de los ciudadanos (jóvenes) de forma significativa.

Por ello, planteamos varios y heterogéneos casos tratados e indagados por diferentes autores en los cuales los MC, abordados desde diversos enfoques significativos, son la piedra angular de sus investigaciones. En ese contexto y mediante los objetivos y metodología ejecutados se ha visto la necesidad e importancia de fundamentar esta investigación con los criterios de los siguientes autores que se presentan a continuación.

Medios y democracia: Blesa-Aledo (2006) expone que las afirmaciones que relacionan a los medios y a la democracia como partes constitutivas y complementarias de un sistema de interacción en el que los medios son servidores públicos, más o menos desinteresadamente, generan un "espacio público” esencial para el intercambio democrático, transmiten valores democráticos u opinión a las audiencias y establecen una "agenda" de temas relevantes para la sociedad que son muy cuestionables actualmente.

Medios para la educación en la escuela: Martínez-Fresneda (2004) explica que los MC transmiten un sistema de valores que presentan una visión sesgada de la realidad. Así también que estos se sirven de realidades muy fácilmente reconocibles por los alumnos. Además, se basan en la actualidad y, mediante ella, introducen las preocupaciones reales y diarias del alumno en la escuela, por lo que se puede decir que los $\mathrm{MC}$ son un espejo donde mirar el acontecer diario, todo lo que sucede a nuestro alrededor. 
Politización mediática de la justicia en Ecuador...

Medios; influencia y poder en elecciones electorales: Díaz (1999) mediante su investigación sostiene cómo los MC en Brasil apoyaron apabullantemente a un candidato en noviembre de 1989.

Medios; contenido transmedia: Majkut y Phil (2011) en su estudio: La Influencia de los medios de comunicación sobre los Contenidos sostienen que el contenido que generan los medios se lo puede llamar "contenido transmedia", debido a que el mismo contenido puede aparecer en varios y diferentes medios. Lo anterior lo explica de la siguiente manera:

Mucha gente sabe que la botella que contiene vino puede influir en el gusto y calidad del mismo, pero pocos se detienen a pensar que un medio de comunicación, el cual contiene un mensaje, posee el mismo poder de alterar el significado contenido en ese medio. (Majkut y Phil, 2011, p. 1)

En resumen, el envase, ya sea una botella o un $\mathrm{MC}$, afecta el contenido que contiene, incluso si uno no reflexiona porque se presta toda la atención al contenido, al vino y no a la botella que entra en juego. Es decir, que cuando este contenido cambia de un medio a otro, algo en él permanece igual y algo se modifica.

Medios; agenda setting: Gómez-Vilchis (2018), en su reciente investigación titulada Medios de comunicación, conocimiento y entendimiento de la opinión pública sobre los sucesos políticos. El caso de México, demuestra el papel de los medios para influir en la importancia que concede la opinión pública a los temas de interés nacional, por medio de la "agenda setting" (teoría de la fijación de la agenda), en la cual los medios tienen una gran influencia sobre el público para establecer cuáles noticias son más relevantes para darles mayor notoriedad y espacio. Ante esto el autor manifiesta que no significa que la opinión pública reaccione de manera pasiva frente a ellos, ya que los efectos de la llamada agenda setting están restringidos por las capacidades de la misma opinión pública.

Medios; construcción de las audiencias: Aguaded y Correa (2004) en su estudio Imagen y construcción de las audiencias: el significado del significado explican cómo los medios de información de masas han generado "otra realidad" en nuestra sociedad, la cual puede llegar a conformar nuestras creencias, actitudes y valores, además de dirigir nuestros comportamientos. 
Sumado a ello, los investigadores sostienen que los medios pueden actuar como poderosos instrumentos capaces de inyectar en la población fuertes dosis de hipnosis colectiva, en el sentido de que generan estados de opinión y también pueden sesgar esa opinión en uno u otro sentido o de quien decide, entre otras técnicas. Además, bajo la influencia de la hipnosis se puede manipular los términos del discurso permisible y establecer los límites de lo "políticamente correcto".

Medios; impacto que generan: Terchila (2014) en su análisis explica que los eventos más importantes internos y externos, sobre los cuales la mayoría de nosotros tenemos muy fuertes opiniones formadas, fueron llevados a nosotros a través de los MC. A partir de aquí la facilidad con la que los medios pueden influir en los juicios de valor y las opciones que la gente elige y las decisiones que pueden tomar después de haberse enfrentado con ellos; decisiones que se generan de acuerdo con los hechos emitidos y presentados en las diferentes noticias que muestran los MC para influenciar en la organización nuestras vidas. La gente depende de los MC para estar informada y los que saben cómo sacar provecho de esta situación ganan un alto por ciento de la audiencia pública. En primer lugar, todas esas formas modernas, modelos de comunicación de masas, actúan sobre la mente humana, probablemente, la televisión es la forma más utilizada de comunicación de masas porque "el público" está conformado por un gran número de personas.

\subsection{Politización mediática de la justicia en Ecuador}

El término "politización de la justicia" ha sido frecuentemente utilizado y duramente cuestionado en la última década en gran parte de América Latina (Ansolabehere, 2005; Boscán, 2010; Guarnieri, Pederzoli y Thomas, 2002). Concretamente, en Ecuador, el sector político se ha visto interferido en las injerencias de la justicia con el propósito de intentar adherirse o someter a las cortes para que los jueces se conviertan en mediadores entre el poder judicial y el poder político; así también, con la intención de buscar mecanismos para accionar de manera indeterminada y arbitraria las decisiones jurídicas. En esta tónica, se ha creído necesario realizar un recorrido por este hecho para lograr comprender de forma efectiva la politización mediática de la justicia en Ecuador. 
Politización mediática de la justicia en Ecuador...

Por eso es necesario analizar lo que nos propone Lucero (2003) al referirse que casi todos los Estados de América han adoptado formalmente formas liberales, representativas de Gobierno que colateralmente repercuten en la justicia. "Al mismo tiempo que muchos países de América Latina fueron sometidos a un retorno a la democracia, y la política basada en la identidad es cada vez más común, es decir, una nueva fuerza también comenzó a dar forma a la ciudadanía: el neoliberalismo" (Warren, 2012, p. 834).

Desde luego, es de suma importancia separar y liberar el poder judicial del Estado y de las influencias que están en su entorno, así como también, de los grupos políticos neoliberales que ejercen presión para dictaminar a su favor. Solo así respetaremos los compromisos sociales con el pueblo, los internacionales con los derechos humanos, entre otros. Sin duda que la inequívoca toma de decisiones, tanto de los grupos de poder como de los MC y los partidos políticos por intervenir en la autonomía de la justicia, está contribuyendo e influyendo a que este organismo judicial se vuelva partidista y se rija según las decisiones neoliberales. En palabras de Jansson y Lindell (2018) esto implica que el espacio social se vuelva aún más estrechamente entretejido que antes.

Hasta aquí hemos intentado reseñar objetivamente; sin embargo, según el vigente Código Orgánico de la Función Judicial del Ecuador registrado en 2009 y sufriendo su última modificación en el año 2015, tenemos que se alude a una normativa judicial integral, que tenga a las personas y colectividades como sujetos centrales de la actuación de las juezas, jueces, fiscales, defensoras y defensores públicos y demás servidores y servidoras judiciales, y que además incorpore los estándares internacionales de derechos humanos y de Administración de Justicia, lo cual es una necesidad impostergable en el Ecuador para la construcción de una sociedad profundamente democrática. En ese sentido, el primer artículo de este Código dice textualmente:

El artículo 1 de esta Constitución vigente establece que el Ecuador es un Estado constitucional de derechos y justicia, por lo que la actuación de servidoras y servidores de la justicia debe responder a los principios y disposiciones constitucionales como una garantía de los derechos, limitación del poder estatal y la realización de la justicia (Código Orgánico de la Función Judicial, 2015) 
Asimismo, en el artículo 8 sobre el Principio de Independencia, determina que:

las juezas y jueces solo están sometidos en el ejercicio de la potestad jurisdiccional a la Constitución, a los instrumentos internacionales de derechos humanos y a la ley. Al ejercerla, son independientes incluso frente a los demás órganos de la Función Judicial. Ninguna función, órgano o autoridad del Estado podrá interferir en el ejercicio de los deberes y atribuciones de la Función Judicial. Toda violación a este principio conllevará responsabilidad administrativa, civil y/o penal, de acuerdo con la ley (Código Orgánico de la Función Judicial, 2015)

Por tanto, se puede evidenciar bajo los artículos del vigente Código que en Ecuador existen los parámetros, leyes y los organismos competentes para vigilar, constatar y no permitir que se incumpla lo contrario. Sin embargo, no cabe duda, que se está incumpliendo la decisión del pueblo que votó a favor de este referéndum. Por esa razón, debemos ser conscientes y acabar, en primer lugar, con la politización mediática que nos genera a diario los $\mathrm{MC}$ y, en segundo lugar, con la politización de la justicia generada por el imperio neoliberal, solo así, buscaremos la fórmula de poseer una justicia parcial e independiente. En síntesis, en palabras de Bond, Diprose y Thomas (2019), lo que urge ahora es una "despolitización" y la "repolitización" de la justicia ecuatoriana.

\subsection{Canal televisivo Ecuavisa}

En Ecuador los MC se han convertido en entes que brindan cobertura mediática en temas sociales, políticos y económicos. Además, tal y como lo señala Sánchez:

A esto se suma el hecho de que al ser los medios empresas mediáticas, tienden también a establecer censuras explícitas o implícitas sobre las diversas notas publicables o no publicables en los mismos, basándose en la relación que existe entre los intereses políticos y económicos de los grupos de poder propietarios o accionistas de un medio de comunicación determinado y la “noticia” en cuestión (Sánchez, 2010, p. 7) 
Politización mediática de la justicia en Ecuador...

Por otra parte, Guillermo Navarro, principal realizador del informe de auditoría de concesiones de frecuencias de radio y televisión en Ecuador (2009), señala en su escrito que entre los objetivos de los medios está el influir en el "statu quo" mediante la afectación del poder mediático, debido a que los grandes medios convierten a sus principales conductores de opinión en voceros políticos. Asimismo, indica mediante un ejemplo ocurrido en Ecuador, cómo los medios mediatizan la información al conllevar una misma línea editorial sobre este tema. Entonces, se puede constatar que en el país existe una monopolización de la información y de los MC, como lo indica Navarro (2009) dentro de su informe, al cual to ha denominado "Concentración Cruzada".

\begin{tabular}{|l|l|}
\hline \multicolumn{2}{|c|}{ Intervalos por rating promedio } \\
\hline \multicolumn{1}{|c|}{ Canal } & $\begin{array}{c}\text { Participación en } \\
\text { la audiencia }\end{array}$ \\
\hline Ecuavisa & $28,02 \%$ \\
\hline Teleamazonas & $27,58 \%$ \\
\hline Gama TV & $15,73 \%$ \\
\hline TC Televisión & $11,14 \%$ \\
\hline $\begin{array}{l}\text { RED } \\
\text { Telesistema }\end{array}$ & $9,13 \%$ \\
\hline Canal UNO & $8,40 \%$ \\
\hline
\end{tabular}

Tabla 1. Cuadro 46. Intervalos por rating promedio. Audiencia total. (Elaboración propia).

Fuente: Comisión de Auditoría Conseciones de Frecuencias de Radio y Televisión, 2009.

Observamos como el canal Ecuavisa concentra la atención de la mayor audiencia en el país, por esa razón, dicho medio puede crear cierto sesgo en su información, además de influenciar a la ciudadanía a través de sus opiniones y contenido para dividir la elipse ecuatoriana. Sin embargo, la pregunta que nos deberíamos plantear los ecuatorianos es: ¿los medios defienden su posición e intereses a través de la mediatización de sus noticias? Ante esto, en la investigación Calidad informativa: credibilidad de medios y periodistas en Ecuador, se sostiene que: 
Los MC han decaído en su credibilidad. El escenario informativo de la crisis mundial y su afectación en Ecuador ha permitido evidenciar dicho deterioro. En muchas de las respuestas este hecho también está imbricado con el contexto político que vive el Ecuador (Mendizábal, 2012, pp. 67-68)

En este contexto, los críticos argumentan que los MC están aumentando a diario la franja de noticias, la cual se ha visto acompañada de la mediatización de intereses comerciales y políticos, dejando de lado ideales, principios y la preocupante ola de información que el público pretende conocer, por lo que se podría sostener que existe concatenación lógica entre profesionales, medios, justicia y política. Así lo indican Odriozola-Chené y Mendizábal (2017) a través de su estudio Hacia un periodismo de calidad en Ecuador: perspectivas de periodistas y audiencia, en donde apuntan que, en cierto sentido, es posible que la transgresión a ciertos indicadores de calidad se constituya en el argumento central de la crítica sectorial mediática que se ha desatado en los últimos tiempos al campo de los MC en Ecuador.

\section{Material y métodos}

La investigación inicia con una intensa revisión bibliográfica digital de artículos científicos tomados, en su totalidad, de las bases de datos: WOS, SCOPUS y DIALNET, así como referencias de revistas ubicadas en los mejores cuartiles de CITESCORE-SJR y JCR.

La metodología utilizada para el presente trabajo de investigación ha sido a través de un diseño de investigación cualitativa, utilizando la metodología del estudio de caso, ya que esta es, quizá, la metodología más idónea para investigar los asuntos que abordan la relación entre los espacios públicos y privados en el contexto de la actividad política y, a la vez, determinar cómo estos cambios políticos se relacionan con la actividad judicial y la actividad social de Ecuador. Son muchos los autores que han utilizado esta técnica en sus estudios de investigación sociológica como lo demuestran los dos sociólogos más importantes del siglo XX Max Weber (1917) y Herbert Spencer (1895). 


\subsection{Muestra:}

Utilizamos un muestreo no probabilístico por conveniencia, pues nos permite seleccionar una muestra de la población más accesible y centrada en el tema de estudio, es decir, los individuos a los que vamos a entrevistar se seleccionan porque están más disponibles para el investigador y son profesionales relacionados con el tema de la investigación, esto sumado junto con los datos obtenidos de la observación no reactiva de nueve noticias de la web de Ecuavisa sobre la politización mediática de la justicia; así, con dichas muestras, se espera obtener los datos necesarios para la realización de un análisis de datos significativo.

\subsection{Instrumentos:}

Se utilizó dos instrumentos para la obtención de datos, en primer lugar, una entrevista semiestructurada con preguntas establecidas con anterioridad de carácter sociodemográfico y exploratorio, pero lo suficientemente flexible como para adaptarse al proceso para obtener unos datos de calidad. Se optó, por otro lado, diseñar una rejilla de análisis de noticias de la web de Ecuavisa creada a partir de la metodología periodística del Fact-checking o comprobación de hechos, es decir, una forma de verificar las afirmaciones fácticas en textos publicados por Ecuavisa en su web sobre la politización mediática de la justicia. Esta rejilla cuenta con una primera columna donde se introduce el titular de la noticia, fuente, fecha de la publicación, link, tags (etiquetas); finalmente, tenemos dos columnas, la primera donde se realiza una descripción cualitativa con base en un análisis explicativo del por qué se incluyó esta noticia y, la otra columna, donde se indica otra fuente probatoria o no de la noticia, es decir, esta misma noticia tratada por otro medio de comunicación.

Seguidamente, se realizó la selección de expertos con formación superior en el grado de doctor y reconocida experiencia en el tema a investigar. Para obtener la guía de validación de expertos se enviaron correos electrónicos a seis doctores académicos con gran trayectoria y reconocida experiencia, los mismos que realizaron las observaciones oportunas, donde es importante destacar la heterogeneidad de las aportaciones, quizás debido al perfil diferenciado que se ha tenido en cuenta al momento de seleccionar los expertos. 
Asimismo, se ha tomado en consideración la gran parte de aportaciones negativas y positivas y las cuales se han incorporado para mejorar el contenido del instrumento acorde con los objetivos planteados de la investigación.

\subsection{Procedimiento:}

Para proceder a realizar la investigación se ha tomado como período de fechas establecidas del 19 al 31 de enero del 2018 para el análisis cualitativo de cada uno de los videos, el mismo que se centra en la politización de la justicia con una orientación de tipo comunicacional-periodístico y que se compone de las siguientes fases o etapas:

1. Análisis de contenido

2. Lenguaje

3. Imagen y contenido visual

4. Feedback - interacción

5. Valores transmitidos

\section{Resultados}

El canal televisivo Ecuavisa es el MC más visto y sintonizado en el Ecuador con el $28.02 \%$ de audiencia, así lo reflejan los datos del informe de concesiones (2009) que nos permiten ver el alto grado (rating promedio) de concentración mediática de los principales canales de televisión existentes en el país, primordialmente, el alcance que en los últimos años ha tenido mayor protagonismo dicho canal. Asimismo, Ecuavisa se ha ganado un prestigio por la evidente información que emite diariamente a la ciudadanía: "es un medio que se ha ganado un prestigio bastante fuerte en el tema informativo de noticias, tiene programas muy buenos, en el ámbito periodístico son los mejores en el país" (Entrevistado 1); su credibilidad, objetividad e imparcialidad así lo amparan: "la trayectoria que tiene Ecuavisa en nuestro país hace que sea un medio que tenga mucha credibilidad, que tenga la confianza de los ecuatorianos" (Entrevistado 2). Es un medio que busca estar donde sucede la noticia, por tanto, está pendiente de lo que se genera en el país política y socialmente hablando. 
Politización mediática de la justicia en Ecuador...

No obstante, Ecuavisa de alguna u otra manera juega un rol importante en la difusión de temas mediáticos para poder generar de algún modo una opinión crítica en su audiencia tal y como lo expone el Entrevistado 1: "hay una línea editorial ahí clara que la manejan, y que de alguna forma influencian al televidente ecuatoriano"; así también, pueden actuar en los procesos que están pendientes de ejecución por el mismo hecho que no ha tenido una gran cobertura mediática: “... hubo una sentencia muy muy rápida porque el tema fue muy mediático, no sé si eso responde tal vez a tu pregunta, el hecho de que son temas mediáticos, temas judiciales que se hacen mediáticos se tratan de una manera mucho más rápida, en ese caso te puedo contestar y decir sí, capaz sí, sí influye, el hecho de que sean mediáticos y la justicia sea más rápida...” (Entrevistado 2 ). Sin duda, como se pudo evidenciar, un medio como Ecuavisa puede repercutir en las democracias para bien o para mal, tomando como punto de partida la concentración mediática de noticias a la ciudadanía y es, precisamente, por ese hecho que nos hemos planteado los siguientes objetivos específicos para determinar el rol mediático, la preservación de intereses y el empoderamiento del pensamiento u opinión de los ciudadanos mediante la influencia mediática de sus informativos.

Primer objetivo: Estudiar el rol mediático de Ecuavisa en la politización de la justicia en Ecuador.

Por tanto, a raíz del análisis se pudo identificar que Ecuavisa en cierta medida está presente en la politización mediática de la justicia en Ecuador a través de la información que emite, así lo evidencia el entrevistado 1: “tal vez no logren influenciar tanto, pero sí pueden ir creando un precepto, algún tipo de pensamiento en el colectivo humano, que los ve, los sigue, por tanto, van a influenciar de alguna forma en la justicia, capaz; no al 100\% pero capaz que si va de alguna forma tomando fuerza en ese sentido". Asimismo, explica que los medios manejan su línea editorial en temas políticos y judiciales. De ahí pasamos a lo referenciado en el trabajo teórico en donde se habló de que los medios aplican la llamada "agenda setting", en la que se da más cobertura a ciertos temas, determinadas personas y a indiscutibles partidos políticos, pues, eso invisibiliza a la ciudadanía y a la imparcialidad de sus noticias, esto lo corrobora el docente en la entrevista de estudio y también en el análisis de contenido del material audiovisual. 
Por su parte, el entrevistado 2 alude que: "cuando hablamos de la politización de la justicia es porque evidentemente alguien interfiere y hace uso de la política a su gusto, si hablamos de estos casos más emblemáticos más mediáticos no sé si ha tenido que ver, porque la verdad, lo que hacemos los periodistas básicamente es escuchar lo que nos dicen"; por tanto, la periodista en ejercicio desde su perspectiva hace hincapié que publicar información no implica una politización de la justicia. Por lo expuesto y después de haber analizado las noticias de dicho canal mediante la rejilla web, se evidenció el rol principal que Ecuavisa le da a la información política, en especial, a los juicios, demandas, temas de corrupción, entre otros, que se están produciendo ahora en contra del anterior Gobierno que presidió el Ec. Rafael Correa Delgado y, por lo cual, se considera que el rol mediático que está alcanzado Ecuavisa hoy en día repercute colateralmente en la politización de la justicia en Ecuador, tal como lo sostienen y apuntan los expertos en la entrevista y basándonos en el análisis cualitativo de la investigación que se compone en: análisis de contenido, lenguaje, imagen y contenido visual, feedback (interacción) $\mathrm{y}$ valores transmitidos.

Segundo objetivo: Explorar el escenario y el ámbito de la información emitida por Ecuavisa en función de sus intereses o la de terceros.

Ante esto, el escenario mediático que protagoniza Ecuavisa a través de sus noticias va en función de una tendencia política, es decir, la información se la puede manejar directa o indirectamente a su favor o a favor de otros, así lo indica el entrevistado 1: “obviamente, pienso que sí, porque desde que ves la agenda de entrevistados que ellos tienen, se nota que están alineados a una línea política, es fácil darse cuenta de eso y siempre creo que se han manejado así... buscan generar algún tipo de opinión en ese sentido. Si pasa en Ecuavisa". Mientras tanto el entrevistado 2 explica que esto es un poco más de perspectivas de los ciudadanos, por consiguiente, sostiene: "no sé si neutral, siempre todos tenemos una postura y asumo yo, que ellos deben tener una postura como $\mathrm{MC}$, pero a veces puede estar mal interpretado el tema de la postura por cómo lo tome la gente". 
Politización mediática de la justicia en Ecuador...

En esta tónica, según lo examinado en los videos, a través del análisis de contenido, lenguaje, imagen y contenido visual y valores transmitidos, se comprobó la notoriedad de ciertos temas políticos en los que el medio brinda y despliega mayor cobertura, tales son los casos de los juicios que en la actualidad se llevan a cabo contra el expresidente Rafael Correa y su grupo de trabajadores que ocuparon diversos cargos en su período. En las fechas establecidas del análisis de los videos, se pudo comprobar que las noticias que más se difundieron ante los oídos de los ecuatorianos fueron: "Fiscalía convoca al expresidente Rafael Correa para que declare en el caso PetroChina..."; "Orden de prisión preventiva para ex ministro...”; "Bloque de Creo en la Asamblea prepara juicio político contra superintendente de Comunicación"; "Expresidente Rafael Correa y ex fiscal Galo Chiriboga serán citados a declarar...”, entre otros. Por lo que se puede contrastar los anteriores titulares con lo dicho por los entrevistados y asegurar que, los medios manejan una línea de lo que se debe cubrir más a fondo y quizá dejar de lado temas que no competen, así como también, que Ecuavisa pertenece a un sector estratégico y por ende defiende sus intereses y la de sus partidos afines. En síntesis, las noticias emitidas por Ecuavisa conllevan un tinte informativo no culpabilizador, pero con cierta influencia en función de sus intereses a pesar de la aparente imparcialidad y objetividad que el medio les da a estas noticias.

Tercer objetivo: Descubrir la intencionalidad e influencia de sus noticias en el empoderamiento del pensamiento u opinión de los ciudadanos.

Por lo que se pudo identificar antes, la información que entrega Ecuavisa se muestra con gran intencionalidad para lograr influenciar mediante su contenido en el empoderamiento del pensamiento u opinión de la ciudadanía, con respecto a eso, el entrevistado 1, destacado en temas audiovisuales y televisión, afirma que:

Obviamente, todo lo que se va a decir en los MC lo dices sabiendo que vas a generar algo en el televidente, no lo dices porque quieres que la gente se entere, lo dices para influenciar en el televidente. Sucede esto, sería raro que un $\mathrm{MC}$ se ponga emitir un tipo de información sin esperar una reacción de la audiencia, espera obviamente una reacción del televidente y en este caso, pues mucho más. 
Asimismo, el entrevistado 2 corrobora lo argumentado: "claro que tienen influencia... evidentemente, han existido opiniones de periodistas en espacio de opinión que tal vez influyen en los ciudadanos". Ante esto y después de analizar profundamente las noticias de la página web del medio, se comprobó que existe una clara evidencia entre la entrevista y lo que difunde Ecuavisa, debido a que las primeras noticias que copan los titulares son las que se relacionan con política y justicia, por lo que se sobreentiende el afán del medio en dar a conocer con frecuencia ante la ciudadanía sobre estos temas en concreto, por un lado, que la gente esté al tanto, como tiene que ser, de los temas más emblemáticos en el acontecer diario, por otra parte, buscan influenciar en el empoderamiento del pensamiento u opinión de los ciudadanos mediante la intencionalidad de sus noticias, es decir, la insistencia con la que se difunden diariamente las mismas hace que las noticias, reales o no, logren en el espectador que suenen y se vean reales; lo dicho se puede corroborar con lo que apunta Romero-Rodríguez y Aguaded (2016) cuando aseguran que los MC son fieles a la fuente, pero con ausencia analítica. En síntesis, al carecer de esto, hace que influencien en el empoderamiento del pensamiento u opinión del televidente.

\section{Conclusiones}

Los lectores se preguntarán ¿es primordial y necesario despolitizar la justicia?, ¿cómo? Los entrevistados coinciden que es necesario la despolitización de la justicia, tal como lo proponen Bond, Diprose y Thomas (2019) lo que urge ahora es una "despolitización" y la "repolitización" de la justicia ecuatoriana. El primer entrevistado propone que sea el propio Gobierno quien genere un aparataje fuerte que los proteja y que les de la libertad de tomar las decisiones que quieran, pero primero tiene que ser el Gobierno el que no quiera esa politización de la justicia. Mientras que el segundo, considera que la función judicial es una sola y tiene que ser independiente como tal, para solucionar el tema, evidentemente, tiene que haber una justicia independiente, por lo cual cree que se deben escoger nuevos jueces, dice: "un nuevo sistema judicial, porque es como más de lo mismo de lo que esta pasando ahora y no llegamos a nada, y siempre ha sido así, el problema es ese, que siempre ha sido así", puntualiza. 
Politización mediática de la justicia en Ecuador...

En resumen, no cabe duda de que es de vital importancia dotar de competencia mediática al público, ya que esto es necesario para revalorar los principios y valores de los ciudadanos, empezando por la formación de una opinión crítica ante este tipo de fenómenos.

Para finalizar, es pertinente resaltar que los hallazgos identificados a partir de este trabajo de investigación trazan un nuevo camino para la construcción de una propuesta seria y concreta, orientada a fortalecer las competencias mediáticas de los ciudadanos ecuatorianos, con el fin de que este tipo de fenómenos no se reproduzcan en los pueblos humildes, soberanos y democráticos.

Es importante destacar que la presente investigación está sujeta a muchas limitaciones por lo que los resultados tienen que interpretarse con precaución, pues, aunque la muestra para una investigación preliminar puede ser representativa, indudablemente, una muestra mayor puede confirmar aún con más rigor las conclusiones de esta investigación. Es decir, se necesitarían más sujetos, una muestra mayor y significativa para poder generalizar los resultados obtenidos.

Otra línea de investigación que se ha descubierto mientras se desarrollaba esta, es la de poder analizar con más profundidad las noticias publicadas en la web de Ecuavisa, haciendo un estudio comparativo con las noticias del mismo tipo que realizan otros medios de Ecuador, esto con el objeto de estudiar si las notas publicadas y su rigor periodístico responden realmente a las noticias o esconden dichas noticias un sesgo en la información que no dan a la ciudadanía. 


\section{Referencias}

Aguaded, J. I. y Correa, R. I. (2004). Imagen y construcción de las audiencias: el significado del significado. Signo y Pensamiento, 23(45), 140-150.

Ansolabehere, K. (2005). Jueces, política y derecho: particularidades y alcances de la politización de la justicia. Isonomía: Revista de Teoría y Filosofia del Derecho, (22), 39-64.

Blesa Aledo, P. S. (2006). Medios de comunicación y democracia: ¿El poder de los medios o los medios al poder? Sphera Pública: Revista de Ciencias Sociales y de la Comunicación, (6), 87-106.

Bond, S., Diprose, G. y Thomas, A. C. (2019). Contesting deep sea oil: Politicisation-depoliticisation-repoliticisation. Environment and Planning C: Politics and Space, 37(3), 519-538. doi:10.1177/2399654418788675

Boscán, G. (2010). Judicialización y politización en América Latina: Una nueva estrategia para el estudio de la interacción entre los poderes públicos. Cuestiones Jurídicas, 4(2), 51-83.

Comisión de Auditoría Concesiones de Frecuencias de Radio y Televisión. (2009). Informe definitivo. Recuperado de https://goo.gl/8KNHqu

Consejo de la Judicatura. (2015). Código Orgánico de la Función Judicial. Recuperado de https://goo.gl/VhzU5D

Díaz, R. E. (1999). Las elecciones venezolanas y la influencia de los medios. Chasqui: Revista Latinoamericana de Comunicación, (65), 60-62.

Downey, J. y Neyazi, T. A. (2014). Complementary and Competitive Logics of Mediatization: Political, Commercial, and Professional Logics in Indian Media. International Journal of Press/ Politics, 19(4), 476-495. doi:10.1177/1940161214545809 
Politización mediática de la justicia en Ecuador...

Ferrero, M. (2009). Violencia y represión en el ocaso de los somoza: las condiciones carcelarias de los presos políticos. Historia Crítica, (39), 154-178.

Gómez Vilchis, R. R. (2018). Medios de comunicación, conocimiento y entendimiento de la opinión pública sobre los sucesos políticos. El caso de México. Revista Mexicana de Opinión Pública, (24), 53-69. doi:10.22201/fcpys.24484911e.2018.24.61516

Guarnieri, C., Pederzoli, P. y Thomas, C. (2002). The Power of Judges: A comparative Study of Courts and Democracy. Estados Unidos: Oxford Socio-Legal Studies.

Haas, E. (2010). Federal tcf: Juridification of Politics-Politization of Justice. Estudios Constitucionales, 8(1), 367-377.

Jansson, A. y Lindell, J. (2018). Media Studies for a Mediatized World: Rethinking Media and Social Space. Media and Communication, 6(2), 1-4. doi:10.17645/mac.v6i2.1495

Lee, S. (2016). The politics of prosecution service reform in new presidential democracies: The South Korea and Russia cases in comparative perspective. Journal of Eurasian Studies, 7(2), 141-150. doi:10.1016/j. euras.2015.09.001

Lucero, J. A. (2003). Locating the "Indian Problem". Community, Nationality, and Contradiction in Ecuadorian Indigenous Politics. Latin American Perspectives, 30(1), 23-48. doi:10.1177/0094582X02239143

Majkut, P. y Phil, C. (2011). La influencia de los medios de comunicación sobre los contenidos. Nuevo Itinerario Revista de Filosofía, (6). Recuperado de http://revistas.unne.edu.ar/index.php/nit/article/view/3196 
Martínez-Fresneda, H. (2004). La influencia de los medios de comunicación en el proceso de aprendizaje. Revista ComunicaR, (22), 183-188. Recuperado de http://www.redalyc.org/pdf/158/15802228.pdf

Medrano, A. (2017). La politización de la sociedad y de la vida. Recuperado de https://goo.gl/qkoqnR

Mendizábal, R. I. (2012). Calidad informativa: Credibilidad de medios y periodistas en el Ecuador. Revista ComHumanitas, 3(3), 53-69. Recuperado de http://comhumanitas.org/index.php/comhumanitas/article/ download $/ 5 / 5$

Navarro, G. (2009, 30 de octubre). La concentración de medios y sus implicaciones políticas éticas y económicas en el Ecuador. Radio Informaremos. Recuperado de https://goo.gl/9VkQtx

Odriozola Chéné, J. y Mendizábal, I. R. (2017). Hacia un periodismo de calidad en Ecuador: perspectivas de periodistas y audiencia. Cuadernos.Info, (41), 175-192.

Real Academia Española. (2018). Diccionario de la Real Academia Española de la Lengua. Recuperado de https://goo.gl/BnfDLM

Sánchez, A. V. (2010, 24 de agosto). La relación entre el tratamiento mediático corporativo de la contra violencia política y su deslegitimación social. FLACSO Ecuador. Recuperado de https://goo.gl/7bxCzG

Spencer, H. (1895). The principles of psychology. New York: Appleton and Company.

Terchila, S. (2014). The Activity of Mass Media Companies in Europe. the Impact Generated in the Economic, Social and Politic Systems. 21st International Economic Conference of Sibiu 2014, Iecs 2014 Prospects of Economic Recovery in a Volatile International Context: Major Obstacles, Initiatives and Projects, 16, 182-189. doi:10.1016/S2212-5671(14)00790-4 
Politización mediática de la justicia en Ecuador...

Towner, T. y Muñoz, C. L. (2016). Boomers versus Millennials: Online Media Influence on Media Performance and Candidate Evaluations. Social Sciences, 5(56), 1-20. doi:10.3390/socsci5040056

Warren, S. D. (2012). Latin American Identity Politics: Redefining Citizenship. Sociology Compass, 6(10),833-844.doi:10.1111/j.1751-9020.2012.00491.x

Weber, M. (1917). El sentido de la neutralidad valorativa de las ciencias sociológicas y económicas. En M. Weber, Ensayos sobre metodología sociológica (pp. 222-269). Buenos Aires: Amorrortu Editores. 